\title{
Portfolio Cost Management in Offshore Software Development Outsourcing Relationships from Vendor's Perspective: a Systematic Literature Review Protocol
}

\author{
Salman Akbar ${ }^{1,4}$, Siffat Ullah Khan ${ }^{2,3,4}$, Asad Ullah Alam ${ }^{4}$, \\ Shahab Uddin Alam ${ }^{4}$ \\ ${ }^{I}$ Department Of Computer Science\& IT, Abdul Wali Khan University Mardan, Khyber Pakhtunkhwa, \\ Pakistan. \\ ${ }^{2}$ Department Of Software Engineering, ${ }^{3}$ Department Of Computer Science \& IT, \\ ${ }^{4}$ Software Engineering Research Group (SERG_UOM), University Of Malakand Pakistan
}

\begin{abstract}
CONTEXT-Offshore software development outsourcing (OSDO) is an important paradigm of global software development. OSDO is a famous business strategy adopted by many organizations in developed countries by outsourcing their software development work to low-wages countries. OSDO offers many benefits to clients organization including high quality software products, authorized and skilled human resources at economical cost. However outsourcing is not a risk free business. Vendor organizations need to address a number of factors for successful outcomes of the outsourcing relationships. Likewise portfolio cost management $(P C M)$ plays an important role in OSDO relationships.

OBJECTIVE: The objective is to identify the critical success factors (CSFs) and critical barriers (CBs) that play a vital role in PCM by reviewing the literature in a systematic way.

METHOD- Systematic Literature Review (SLR) is based on a structured protocol, and is therefore, different from ordinary literature review. SLR provides in-depth, more thorough and comparatively unbiased results than ordinary literature review.

EXPECTED OUTCOME- We have developed a SLR protocol and in the process of implementation. Our research outcome will be a CSFs and CBs in PCM in the context of OSDO relationships.
\end{abstract}

Keywords: Systematic Literature Review; Portfolio Cost Management; Software Development Outsourcing; Client-Vendor Relationships.

\section{Introduction}

Offshore software development outsourcing (OSDO) is a rapid growing business paradigm in global software development. Although software outsourcing has been accepted and famous business practice for over the last two decades it has shown dramatic increase in recent years and has been the engine of growth and the soul of the software and computer services sector [1]. Software development outsourcing is a contract-based relationship between client and vendor organizations in which a client(s) contracts out all or part of its software development activities to a vendor(s), who provides agreed services in return for remuneration [2].Many organizations in the developed countries are outsourcing their software development projects to vendors at offshore locations to gain low-cost advantage and high quality software production. Our previous research shows that outsourcing gained a dramatic increase after 2001 and is still growing continuously due to economic downturn. Countries such as India, Ireland, China and Russia are the major outsourcing destinations with an increasing offshore outsourcing presence. These countries mostly get software outsourcing work from client companies in the USA, Japan, Australia and the European countries. India's share of the total offshore outsourcing services is significantly larger than any other country [3]. The other emerging countries are China, Russia, Mexico and Ireland. It is predict that China will become the biggest competitor to India in offshore outsourcing and may also overtake India in offshore outsourcing share by 2015 [4]. But there is a need to understand that why some services are produced in house and why others are contracted out [4]. Client organizations outsource their work offshore when they expect the market to be more cost efficient or when they believe skills, knowledge or experience are available via the market that are not available in-house [5]. OSDO is not a risk free business; however it also drew tending due to complexity and high quality software development. The most critical success factor in OSDO is to manage portfolio project cost management. Portfolio management based on an overall performance of the project set. To decide whether to bid for a contract, software cost estimation can provide a condition in a portfolio management [6]. Portfolio management is a process in which projects are evaluated, selected, prioritized, launched (i.e., introduced to the public or to a market), and canceled [7]. The process for achieving balanced resource allocation in terms of value maximization, strategic alignment, the risk level, and the number of ongoing projects is discussed in the 
literature on managing new product development and is referred to as portfolio management of new product development projects, or portfolio management for short [8]. IT PM is a project evaluation and prioritization process that drives organizations to take a balanced approach to evaluating individual projects and to optimize the mix of projects it undertakes [9]. Effective product portfolio management is a continuous process for defining, evaluating and prioritizing the set of current and planned product development activities [9]. PPM considers the entire portfolio of projects a company is engaged in, in order to make decisions in terms of which projects are to be given priority, and which projects are to be added to or removed from the portfolio [10]. Portfolio management is the management of organization assets similar to financial performance of the portfolio by balancing risk and return.

The main objective of this research is to gain an in-depth and more thoroughly understanding of portfolio cost management in the context of OSDO relationships from vendor's perspective. The expected outcomes of the review are the identification of critical success factors (CSFs) and Critical barriers (CBs) to be addressed by vendor organizations for enhancing PCM in OSDO relationships.

RQ1: What are the critical success factors, to be addressed by vendor organizations that influence/assist the portfolio cost management in the context of small size vendor organization in offshore software development outsourcing relationships?

RQ2: What are the critical barriers, faced by small size vendor organizations, in portfolio cost management in the context of small size vendor organization in offshore software development outsourcing relationships?

\section{Background}

Offshore software development outsourcing (OSDO) is a rapid growing business paradigm in global software development. Outsourcing is a contract based business where the developed countries outsource their software to low wages countries in developing countries for remuneration [2]. Outsourcings relationships have many business strategies embedded but the most important is offshore software outsourcing. Mostly, bundle of projects outsourced to vendor's organizations from client's countries at the same time is called portfolio software projects. However, the management of portfolio projects is too difficult for vendor's organization particularly for the small size vendor's organizations. The concept of portfolio management originates from the context of large organizations, where activities are primarily organized as projects; there is an explicit strategy, and dedicated portfolio management personnel exist [11]. Project portfolio management (PPM) has largely developed around the following elements: providing a centralized view of all the projects in an organization, enabling a financial and risk analysis of projects, modeling interdependencies between a family of projects, incorporating constraints on resources shared between projects, enabling prioritization and selection of projects, ensuring accountability and governance at the portfolio level, allowing for portfolio optimization and providing support in the form of standardized processes and software tools [12]. Most organizations struggle to demonstrate business gains from investments in information technology. New research reveals how to meet the challenges of IT portfolio management to deliver tangible results [9]. Successful portfolio management achieves a balance between the four potentially conflicting goals of 1) maximizing the financial value of the portfolio, 2) linking the portfolio to strategy, 3) balancing it on relevant dimensions, and 4) ensuring that the total number of ongoing activities is feasible. While portfolio management is essentially about resource allocation, the inherent complexity of the issues involved keeps it far from being a mechanistic exercise [8].There are many factors and hurdle that effects project managers in effective management of portfolio projects. These barriers are time management and knowledge sharing management. However, the most important success factor in portfolio projects is the portfolio cost management. Portfolio management proposed by Fewster focuses on adjusting only deadlines and the number of employees. However, effective project management should focus on people, product, process and project not only one [13]. The need to extend the traditional single-project management approach to the management of sets of projects or project portfolios became apparent decades ago and greatly influences recent research directions [14]. Portfolio management increases flexibility in reducing project risks, experiments is performed to confirm this assumption. The results can guide managers in adopting a portfolio viewpoint in their project management [6].

A number of researchers have tried to address some of the issues of portfolio project management in global software development outsourcing.

Harry Markowitz study they articulated the basis for the modern portfolio theory (MPT). Datz provides [12] mentioned the main benefits of PPM and should expect from adopting PPM approaches. These include: (1) maximizing the value of IT investments while minimizing risk, (2) improving communication and alignment between IT and business leaders, and (3) encouraging business leaders to act as team players, allowing planners to allocate resources more efficiently and to terminate projects. The modern portfolio theory allows determining the specific mix of investments generating the highest return for a given level of risk. Another modern approach portfolio management, projects are evaluated, selected, prioritized, launched (i.e., introduced to the public or to 
a market), and canceled [8]. PPM considers the entire portfolio of projects a company is engaged in, in order to make decisions in terms of which projects are to be given priority, and which projects are to be added to or removed from the portfolio [10]. Leffingwell mentioned a very high-level view to portfolio management level; the company's executives define investment themes that drive the resource allocation and thus investment priorities of the company [15].

\section{Systematic Literature Review Protocol}

This paper describes the SLR protocol for PCM in offshore software outsourcing relationships from vendors' perspective in a systematic way. Kitchenham [16] describes the three main phases of a systematic review process. These are: planning the review, conducting the review, and reporting the review. In this paper we describe the first phase of a systematic review (i.e. planning the review). The outcome of this phase is a systematic review protocol that specifies the purpose and procedures for the review. It is argued that a predefined protocol is necessary to reduce the possibility of researcher's bias.

\subsection{Constructing Search String(S)}

The following detail will help in designing a search strings relevant to our research questions.

\section{Population:}

Offshore software development outsourcing vendors organizations

\section{Intervention:}

Factors and characteristics

\section{Outcome of relevance:}

Best practices for portfolio cost management in the context of global software development outsourcing relationships, innovation in portfolio cost management.

\section{Experimental Design:} above detail is:

Exploratory study, case study, experts' opinions. An example of the research question containing the

RQ1: [What factor/ characteristics] "INTERVENTION" to be addressed by vendor organizations for [Enhancement of Portfolio Cost Management] "OUTCOME OF RELIVENCE" in the context of [Global Software Development Outsourcing Relationships] "POPULATION".

\subsection{Search strategy:}

\subsection{Identifying Search Terms:}

The following search strategy is used for the formulation of search terms/strings.

a) Use the research questions(RQs) for the derivation of major terms, by identifying Population, intervention and outcome;

b) For these major terms, find the alternative spellings and synonyms;

c) Verify the key words in any relevant paper;

d) Use of Boolean operators for conjunction if the database allows, in such a way, to use 'OR' operator for the concatenation of alternative spellings and synonyms whereas 'AND' for the concatenation of major terms

e) The modified/summarized form of the search strings for the research questions, if Required The fifth step is included from the following SLRs [17][18][19][20].

\section{Results for a)}

RQ1: Global software development outsourcing relationships, vendor organizations, factors influencing portfolio cost management.

RQ2: Portfolio cost management, barriers, vendors, global software development outsourcing relationships.

\section{Results for b)}

RQ1: Project portfolio cost management: ("project portfolio cost management" OR "project portfolio price management" OR "project portfolio charge management" OR "project portfolio rate management "OR "project portfolio fee management" OR "project portfolio budget management" OR "project portfolio outlay management" OR "project portfolio expense management" OR "project portfolio expenditure management") Global software development: ("global software development" OR "information system outsourcing" OR "information technology outsourcing" OR "IS outsourcing" OR "IT outsourcing" OR "CBIS outsourcing" OR 
"computer based information system outsourcing" OR "software contracting-out" OR "distributed software development" OR "multi-site software development")

Vendor(s): ("vendor" OR "vendors" OR "service-provider" OR "dealer" OR "trader" OR "marketer" OR "seller" OR "developer", "businessperson" OR "hawker" OR "huckster" OR "peddler" OR "traveling salesperson")

Factors: ("factors" OR "drivers" OR "motivators" OR "elements "OR "characteristics" OR "parameters")

Negative impact: ("negative impact" OR "relationships failure" OR "poor results" OR "dissatisfaction" OR "disappointment" OR "displeasure" OR "disagreement" OR "bad effect" OR "lack of trust" OR "unconfident" OR "rejection" OR "uncertain decision" OR "conflict" OR "uncertainties"

RQ2: Project portfolio cost management: ("project portfolio cost management" OR "project portfolio price management" OR "project portfolio charge management" OR "project portfolio rate management" OR "project portfolio fee management" OR "project portfolio budget management" OR "project portfolio outlay management" OR "project portfolio expense management" OR "project portfolio expenditure management")

Barriers: ("barriers" OR "barrier" OR "obstacles" OR "hurdles" OR "risks" OR "risk analysis" OR "critical factors" OR "barricade" OR "railing" OR "bar" OR "blockade" OR "bound" OR "boundary" OR "confines" OR "curtain" OR "ditch" OR "enclosure" OR "fence" OR "fortification" OR "gully" OR "hurdle" OR "impediment" OR "limit" OR "palisade" OR "railing" OR "rampart" OR "stop" OR "trench")

Vendor(s): ("vendor" OR "vendors" OR "service-provider" OR "dealer" OR "trader" OR "marketer" OR "seller" OR "developer", "businessperson" OR "hawker" OR "huckster" OR "peddler" OR "traveling salesperson")

Global software development: ("global software development" OR "information system outsourcing" OR "information technology outsourcing" OR "IS outsourcing" OR "IT outsourcing" OR "CBIS outsourcing" OR "computer based information system outsourcing" OR "software contracting-out" OR "distributed software development" OR "multi-site software development")

\section{Results for c)}

Project portfolio cost management in software outsourcing relationships, barriers, software outsourcing vendors, global software development outsourcing relationships.

\section{Results for d)}

RQ1: ("Project portfolio cost management" OR "project portfolio price management" OR "project portfolio charge management" OR "project portfolio rate management" OR "project portfolio fee management" OR "project portfolio budget management" OR "project portfolio outlay management" OR "project portfolio expense management" OR "project portfolio expenditure management")AND (“"Global software development" OR "information system outsourcing" OR "information technology outsourcing" OR "IS outsourcing" OR "IT outsourcing" OR "CBIS outsourcing" OR "computer based information system outsourcing" OR "software contracting-out" OR " distributed software development" OR "multi-site software development") OR ("factors" OR "drivers" OR "motivators" OR "elements" OR "characteristics" OR "parameters") OR ("vendor" OR "vendors" OR "service-provider" OR "dealer" OR "trader" OR "marketer" OR "seller" OR "developer", "businessperson" OR "hawker" OR "huckster" OR "peddler" OR "traveling salesperson") OR ("negative impact" OR "relationships failure" OR "poor results" OR "dissatisfaction" OR "disappointment" OR "displeasure" OR "disagreement" OR "bad effect" OR "lack of trust" OR "unconfident" OR "rejection" OR "uncertain decision" OR "conflict" OR "uncertainties"))

RQ2: ("Project portfolio cost management" OR "project portfolio price management" OR "project portfolio charge management" OR "project portfolio rate management" OR "project portfolio fee management" OR "project portfolio budget management" OR "project portfolio outlay management" OR "project portfolio expense management" OR "project portfolio expenditure management")AND (“"Global software development" OR "information system outsourcing" OR "information technology outsourcing" OR "IS outsourcing" OR "IT outsourcing" OR "CBIS outsourcing" OR "computer based information system outsourcing" OR "software contracting-out" OR " distributed software development" OR "multi-site software development") OR ("factors" OR "drivers" OR "motivators" OR "elements" OR "characteristics" OR "parameters") OR ("vendor" OR "vendors" OR "service-provider" OR “dealer" OR "trader" OR "marketer" OR "seller" OR "developer", "businessperson" OR "hawker" OR "huckster" OR "peddler" OR "traveling salesperson") OR ("negative impact" OR "relationships failure" OR "poor results" OR "dissatisfaction" OR "disappointment" OR "displeasure" OR "disagreement" OR "bad effect" OR "lack of trust" OR "unconfident" OR "rejection" OR "uncertain decision" OR "conflict" OR "uncertainties")). 


\section{Result for e)}

((“Project portfolio cost management" OR "project portfolio price management" OR "project portfolio charge management" OR "project portfolio rate management" OR "project portfolio fee management" OR "project portfolio budget management" OR "project portfolio outlay management" OR "project portfolio expense management" OR "project portfolio expenditure management") AND ("Global software development" OR "information system outsourcing" OR "information technology outsourcing" OR "IS outsourcing" OR "IT outsourcing" OR "CBIS outsourcing" OR "computer based information system outsourcing" OR "software contracting-out" OR "distributed software development" OR "multi-site software development"))

\section{Search term break-up}

We will first use the search strings RQ1 and RQ2 mentioned in (d) as our search terms. As some of the databases don't allow the lengthy search strings we will split the search term into smaller sub strings and will do separate search for each of these search strings. Finally we will summarize the search results by removing the redundancy. It should be noted that while using the IEEEXplore digital library, the search strings mentioned in (d) will be used with a technique that it should be typed/copied in the window pane (available below the textboxes on the page) instead of text-boxes provided in the advance search option of the digital library. This tip was identified/learned from our previous reviews experiences.

\section{String-1}

("Project portfolio cost management" OR "project portfolio price management" OR "project portfolio charge management" OR "project portfolio rate management" OR "project portfolio fee management" OR "project portfolio budget management" OR "project portfolio outlay management" OR "project portfolio expense management" OR "project portfolio expenditure management")AND ("Global software development" OR "information system outsourcing" OR "information technology outsourcing" OR "IS outsourcing" OR "IT outsourcing" OR "CBIS outsourcing" OR "computer based information system outsourcing" OR "software contracting-out" OR "distributed software development" OR "multi-site software development")

\section{String-2}

("Project portfolio cost management" OR "project portfolio price management" OR "project portfolio charge management" OR "project portfolio rate management" OR "project portfolio fee management" OR "project portfolio budget management" OR "project portfolio outlay management" OR "project portfolio expense management" OR "project portfolio expenditure management")AND ("Factors" OR "drivers" OR "motivators" OR "elements" OR "characteristics" OR "parameters")

\section{String-3}

("Project portfolio cost management" OR "project portfolio price management" OR "project portfolio charge management" OR "project portfolio rate management" OR "project portfolio fee management" OR "project portfolio budget management" OR "project portfolio outlay management" OR "project portfolio expense management" OR "project portfolio expenditure management")AND ("barriers" OR "barrier" OR "obstacles" OR "hurdles" OR "risks" OR "risk analysis" OR "critical factors" OR "barricade" OR "railing" OR "bar" OR "blockade" OR "bound" OR "boundary" OR "confines" OR "curtain" OR "ditch" OR "enclosure" OR "fence" OR "fortification" OR "gully" OR "hurdle" OR “impediment" OR "limit" OR "palisade" OR "railing" OR "rampart" OR "stop" OR "trench")

\section{String-4}

("Project portfolio cost management" OR "project portfolio price management" OR "project portfolio charge management" OR "project portfolio rate management" OR "project portfolio fee management" OR "project portfolio budget management" OR "project portfolio outlay management" OR "project portfolio expense management" OR "project portfolio expenditure management")AND ("vendor" OR "vendors" OR "service-provider" OR “dealer" OR “trader" OR “marketer" OR “seller” OR “developer”, "businessperson” OR "hawker" OR "huckster" OR "peddler" OR “traveling salesperson").

\subsection{Resources to be searched}

IEEEXplore, Science Direct, Cite Seer digital library, ACM Portal, Google Scholar and Springer Link.

\subsection{Search Constraints and Validation}

3.6. e will search all published papers related to our research questions and will not put any date boundaries. A prior search was conducted using the following set of major terms and we found certain relevant papers in ACM and IEEEXplore digital libraries. 
("Project portfolio cost management" OR "project portfolio price management" OR "project portfolio charge management" OR "project portfolio rate management" OR "project portfolio fee management" OR "project portfolio budget management" OR "project portfolio outlay management" OR "project portfolio expense management" OR "project portfolio expenditure management") AND ("Global software development" OR "information system outsourcing" OR "information technology outsourcing" OR "IS outsourcing" OR "IT outsourcing" OR "CBIS outsourcing")

The papers, identified through this string, were used for the validation of our final search strings.

\subsection{Search documentation}

The candidate papers will be stored in the form of a table with keeping following records: (S. No, Name of Database, Search Strategy, Search Phase, Date of Search, Years Covered, No of Publication Found, Initial Selection Decision, Final Selection Decision

\section{Publication Selection}

\subsection{Inclusion Criteria}

The inclusion criteria we used to determine which piece of literature (papers, technical reports, or 'grey literature' etc.) found by the search string (s) will be used for the data extraction. We will only consider papers related to global software development outsourcing with focus on project portfolio cost management that are written in English. The criteria are listed below:

- Studies that describe factors/motivators for portfolio cost management in global software development outsourcing

- Studies that describe portfolio cost management from vendors' perspective

- Studies that describe global software development outsourcing relationships with the focus on portfolio cost management

- Studies that describe criteria for a successful portfolio cost management

- Studies that describe issues/barriers in portfolio cost management

- Studies that describe factors affecting the continuation/termination of portfolio cost management.

\subsection{Exclusion Criteria}

This section describes the exclusion criteria in order to decide which piece of literature found by the search term will be excluded/ignored. The criteria are listed below:

- Studies that are not relevant to the research questions

- Studies that don't describe global software development outsourcing portfolio cost management

- Studies that don't describe global software development outsourcing relationships with the focus on portfolio cost management

- Studies other than global software development outsourcing relationships.

\subsection{Selecting Primary Sources}

Initial selection of the primary sources will be performed by reviewing the title, keywords and abstract. The purpose is to exclude/ignore only those results which have no relevance to the problem/research questions. The primary sources chosen in the initial selection process will be checked against the aforementioned inclusion/exclusion criteria by reviewing carefully through full text of the studies. The source will be sent to the secondary reviewer, for review in case of any uncertainty regarding the inclusion or exclusion decision. In order to control researcher's bias, the secondary reviewer will be approached for guidance and validation of the publication selection process. The record of inclusion/exclusion decision regarding each primary source will be sustained properly. This will describe the reasons/justification whether or not the primary source has been included in the final review.

\section{Publication Quality Assessment}

The measurement of quality is performed after final selection of publications. The quality of publications is assessed in parallel at the time of data extraction. The quality checklist contains the following questions:

- Is it clear how portfolio cost management was measured/evaluated in global software development outsourcing relationships?

- Is it clear how the CSFs (critical success factors) for portfolio cost management between clients and vendors were identified in global software outsourcing relationships?

- Is it clear how the CBFs (critical barrier factors) for portfolio cost management between clients and vendors were identified in global software outsourcing relationships.

Each of the above questions will be marked/answered as 'YES' or 'NO' or 'Partial' or 'N.A'. A secondary reviewer will score a small subset for validation. 


\section{Data Extraction Strategy}

The data extraction phase will be undertaken by a single reviewer, who will alone be responsible for the data extracted. A secondary reviewer will be approached for guidance in case of an issue regarding the data extraction. The inter-rater reliability test will be performed after the data extraction process by the primary reviewer. The secondary reviewer will select few publications randomly from the list of publication already chosen by the primary reviewer. The secondary reviewer will independently extract the data from the randomly selected publication. The results will then be compared with the results produced by the primary reviewer.

Primary Reviewer: Salman Akbar Secondary Reviewers: Dr Siffat Ullah Khan and Asad Ullah Alam

\section{Data Synthesis}

Due to two research questions, the synthesis will also be categorized into two parts. For the research question1, the data will be synthesized by creating one summary table having the columns (S.No, CSF's, Frequency, Percentages) showing the list of all the CSFs along with their frequencies and percentages. The complete detail of every CSF mentioned in the Summary table will be recorded in a separate table which will hold the following the columns (CSF group name, S.No of reference, CSF subgroups, paper reference/paper title). For the research question2, the same process will be performed as for the RQ1 mentioned above. The statistical analysis will be performed using SPSS.

\section{Acknowledgment}

We are thankful to Software Engineering Research Group at University of Malakand (SERG_UOM) for the review and their valuable comments in validation process of the protocol. SERG_UOM was founded/established by Dr Siffat Ullah Khan in January, 2011.

\section{References}

[1] M. Miozzo and D. Grimshaw, "Modularity and innovation in knowledge-intensive business services: IT outsourcing in Germany and the UK," Research Policy, vol. 34 (2005), pp. 1419-1439, 2005.

[2] S. Khan, M. Niazi, and R. Ahmad, "Factors influencing clients in the selection of offshore software outsourcing vendors: an exploratory study using a systematic literature review," Journal of systems and software, vol. 84(4), pp. 686-699, 2011.

[3] S. Khan, M. Niazi, and R. Ahmad, "Critical Success Factors for Offshore Software Development Outsourcing Vendors: A Systematic Literature Review," presented at The 4th IEEE International Conference on Global Software Engineering, Ireland, 2009.

[4] M. D. Minevich and F. Richter, "Global outsourcing report," CIO Insight, pp. 1-48, 2005.

[5] S. Betz and A. Oberweis, "Knowledge Transfer in IT Offshore Outsourcing Projects: An Analysis of the Current State and Best Practices," International Conference on Global Software Engineering, vol. .45, pp. 978-0-7695-4122-8/10, 2010.

[6] W. Jiamthubthugsin and D. Sutivong, "Portfolio Management of Software Development Projects Using COCOMO II," presented at ICSE, shanghai, China, 2006.

[7] R. G. Cooper, "Product Leadership: Creating and Launching Superior New Products," Reading, MA: Perseus Books, 1998.

[8] J. Vahaniitty, K. Rautiainen, and C. Lassenius, "Small software organizations need explicit project portfolio management," International Business Machines Corporation, vol. 54(2), 2010.

[9] M. Jeffery and I. Leliveld, "Best practices in IT portfolio management," Sloan Manage. Rev, vol. 45(3), pp. 41-49, 2004.

[10] M. Lycett, A. Rassau, and J. Danson, "Programme management: acritical review," Int J ProjManag, vol. 22(4), pp. 289-299, 2004.

[11] M. Martinsuo, "BProject portfolio management: Contingencies, implementation and strategic renewal," presented at in Project Portfolio Management Strategic Management Through Projects., Helsinki, Finland, 2001.

[12] D. B. Reyck, G. Y. Cockayne, M. Lockett, R. S. Calderini, M. Moura, and A. Sloper, "The impact of project portfolio management on information technology projects," International Journal of Project Management, vol. 23 (2005), pp. 524-537, 2005.

[13] R. S. Pressman, "Software Engineering: A Practitioner's Approach," McGraw-Hill, 2005.

[14] R. Gareis, " Management by projects organization, structure and control," International Journal o/Pmject Management, vol. 7(4), 1989.

[15] D. Leffingwell, "Scaling Software Agility: Best Practices for Large Enterprises: USA," Addison Wesley, 2007.

[16] B. Kitchenham, "Guidelines for performing Systematic Literature Reviews in Software Engineering," vol. EBSE-TechnicalReport, 2007.

[17] A. Alam, U and S. Khan, U, "Knowledge Sharing Management in Offshore Software Development Outsourcing Relationships from Vendors' Perspective: A Systematic Literature Review Protocol," presented at The 5th IEEE International Conference on Software Engineering, Malaysia, 2011.

[18] Muhib Ullah, Siffat Ullah Khan, "Challenges and Success Factors in Mobile Commerce Customer Relationships Management: A Systematic Literature Review Protocol" IOSR Journal of Computer Engineering (IOSRJCE), Volume 2, Issue 3 (July-Aug. 2012), PP 25-34

[19] Muhammad Salam, Dr.Siffat Ullah Khan, "Software Development Multi-Sourcing Relationship Management Model (Sdmrmm) Protocol (A Systematic Literature Review)", IOSR Journal of Computer Engineering (IOSRJCE) ISSN: 2278-0661 Volume 3, Issue 2 (July-Aug. 2012), PP 24-31

[20] Abdul Wahid Khan, Siffat Ullah Khan,"Offshore Software Development Outsourcing Contract from Vendors' Perspective: A Systematic Literature Review Protocol", IOSR Journal of Computer Engineering (IOSRJCE) ISSN: 2278-0661 Volume 2, Issue 4 (July-Aug. 2012), PP 26-37 